Address for Correspondence: Rebecca S. Bahn, MD, Professor of Medicine, Mayo Clinic College of Medicine, Rochester, MN 55905, USA. Email: bahn.rebecca@mayo.edu

\begin{tabular}{|l|}
\hline Access this article online \\
\hline $\begin{array}{l}\text { Website: } \\
\text { www.intern-med.com }\end{array}$ \\
\hline DOI: \\
10.1515/jtim-2016-0015 \\
\hline Quick Response Code: \\
\hline \\
\\
\\
口) \\
口) \\
\hline
\end{tabular}

\title{
Graves' disease syndrome: A need for unified therapy
}

\author{
Rebecca S. Bahn \\ Mayo Clinic College of Medicine, Rochester, MN 55905, USA
}

Graves' disease (GD) can be thought of as a syndrome with variable expression of its clinical manifestations. The hyperthyroidism of GD has a prevalence in the United States of about 1\% (3 million people) and a prevalence of 50-80/100,000 women/yr and 8/100,000 men/yr. Graves' ophthalmopathy or orbitopathy (GO) is an autoimmune orbital disease that can be diagnosed in some $30-50 \%$ of patients with $\mathrm{GD}^{[1]}$. The annual age-adjusted incidence rate of GO is $16 / 100,000$ women and $3 / 100,000$ men $^{[2]}$. About $7 \%$ of patients with GO have the associated pretibial skin involvement termed thyroid dermopathy and thyroid acropachy is even more rare (Figure 1). While therapy exists for each of the clinical manifestations of GD, none is uniformly successful or without the risk for significant side effects. In this commentary, I will review the benefits and disadvantages of each of the available treatment options for hyperthyroidism and GO and discuss the need for novel therapy addressing the pathophysiology of the GD syndrome.

Graves' hyperthyroidism is generally treated with radioactive iodine (RAI) ablation of the thyroid, antithyroid medication (usually methimazole; MMI), thyroidectomy, or a combination of these approaches ${ }^{[3]}$. The therapeutic goal of both surgery and RAI is to render the patient hypothyroid which necessitates lifelong thyroid hormone replacement with laboratory monitoring. While thyroidectomy alleviates hyperthyroidism rapidly following adequate surgical preparation, there exists the potential for complications, including recurrent laryngeal nerve damage or hypoparathyroidism. Therefore, this therapeutic option depends upon the availability of a high-volume thyroid surgeon. Patients undergoing RAI avoid the need for surgery. However, this therapy may not be effective for several weeks to months and patients may rarely require an additional dose. In addition, there exists a small but real risk for GO worsening following RAI therapy in patients with active eye disease, especially smokers. Therefore, glucocorticoid (GC) administration for several weeks following RAI administration, or choice of another treatment modality, is recommended ${ }^{[4]}$. Because a low thyroid hormone level increases the risk for development or worsening of GO, early replacement of thyroid hormone with appropriate dose titration is important following either thyroidectomy or RAI. In contrast, euthyroidism can often be achieved within a few weeks in patients taking MMI and some patients may enter remission while on this medication. However, others are very difficult to manage and require frequent laboratory assessment and dose adjustments. MMI is generally administered for only about 12-18 months owing to the potential side-effects of agranulocytosis and hepatotoxicity. Following discontinuation of MMI, the majority of patients experience recurrent hyperthyroidism and require definitive treatment with either thyroidectomy or RAI. Owing to the rare occurrence of hepatic necrosis, the antithyroid drug propylthiouracil is no longer used except in a few circumstances including early pregnancy ${ }^{[3]}$.

Most patients with GO experience have only mild eye redness or discomfort. However, about 20\% develop more serious complications, including forward displacement of the eyes (proptosis), diplopia, or significant edema around the 


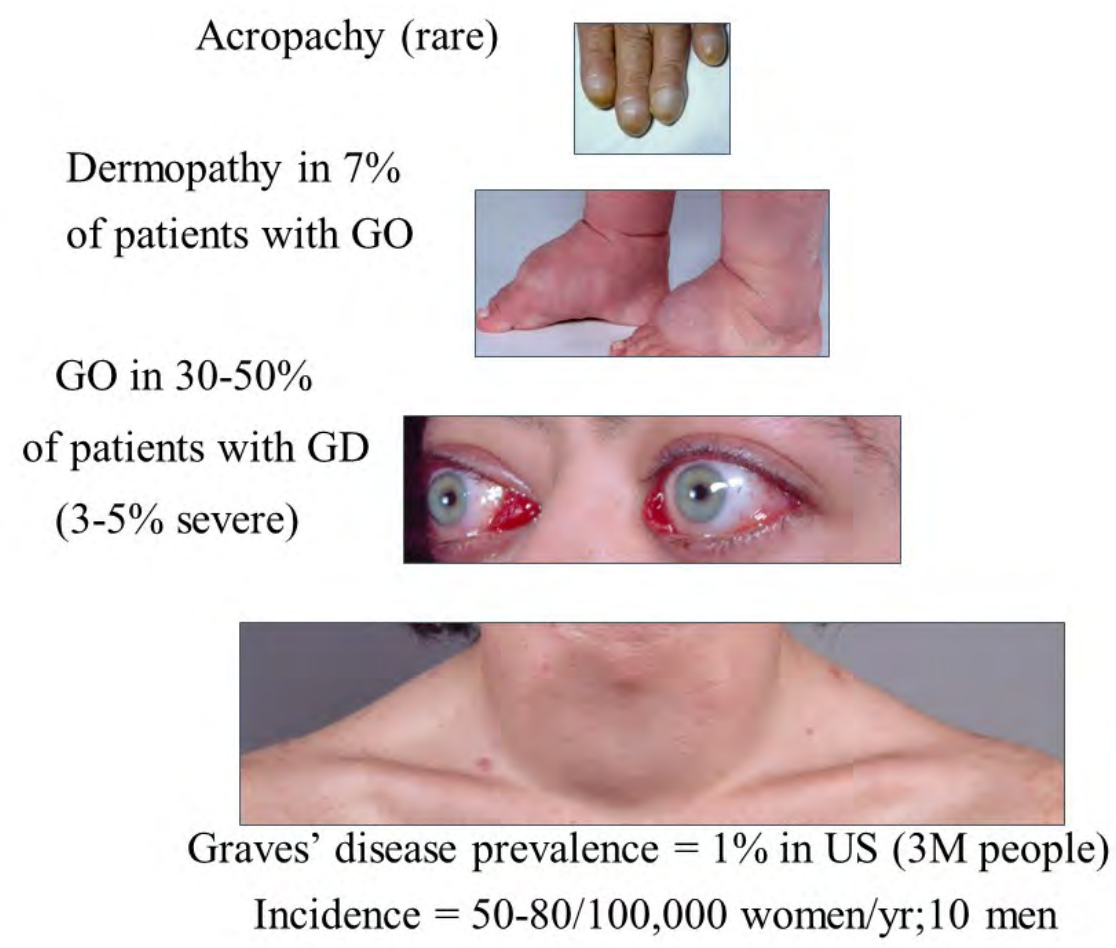

Figure 1: The pyramidal structure of the Graves' disease syndrome

eyes. Some 3-5\% develop intense pain with severe edema or even loss of vision. The available treatments for GO include intravenous and oral corticosteroids, orbital irradiation, and orbital decompression surgery ${ }^{[4]}$. Owing to the potentially significant side effects inherent in these therapies, patients with mild disease are generally treated only symptomatically using ocular lubrication with artificial tears or gels applied at bedtime. In addition, smoking cessation is crucial as smoking is the most important GO risk factor yet identified.

Immunomodulatory therapy is appropriate for patients with active GO and moderate to severe disease. Glucocorticoids (GC) given orally were long considered the mainstay of management at starting doses of prednisone generally range between $40-100 \mathrm{mg} /$ day with tapering over 10-24 weeks (cumulative dose of 2-6 grams). However, more recent evidence from several prospective clinical trials suggests that GC given by the intravenous (IV) route is more effective (generally methylprednisolone $500 \mathrm{mg}$ weekly x 6 weeks, followed by $250 \mathrm{mg}$ weekly x 6 weeks for a total dose of $4.5 \mathrm{~g})^{[5]}$. However, while some $50-70 \%$ of patients improve following a course of IVGC, about $20 \%$ relapse and disthyroid optic neuropathy is not prevented in $7 \%$. Compared with oral GC therapy, IVGC is associated with fewer and less significant adverse events, improved quality of life and less need for subsequent ocular surgery. Although severe hepatotoxicity is a rare complication of IVGC therapy, it appears to occur only in patients receiving a cumulative dose $>8 \mathrm{~g}$ of methylprednisolone. The use of orbital irradiation has declined in recent years owing to its limited utility, except in the treatment of extraocular muscle impairment and in combination with other therapeutic modalities. The risk of radiation retinitis exists, even in patients who receive appropriate radiation dose and delivery.

Rituximab (RTX) is a targeted biological agent (anti-CD20 chimeric monoclonal antibody) that blocks early B cell activation and differentiation and induces transient B-cell depletion. Recently 2 small double-masked randomized controlled trials of rituximab (RTX) treatment in patients with moderately severe and active GO have been reported ${ }^{[6,7]}$. However, results of these studies were quite disparate. In the study by Stan and colleagues $(n=25$ patients), RTX was given as 2 infusions (1000 $\mathrm{mg}$ each) or two saline infusions at 2 week intervals. Both patient groups improved similarly over the course of the study; no differences were noted at either 24 or 52 weeks in any disease parameter. The disease inactivation rate at 24 weeks was 31\% in the RTX group and $17 \%$ in the placebo group $(P=0.41)$. Further, there were 5 moderately severe adverse advents in the RTX group (1 in the control group), including 2 patients who developed dysthyroid optic neuropathy and events the complications encountered in the RTX-treated patients were significant with 5 ( 1 in 
the control group) including 2 patients who developed optic neuropathy and 1 with a significant lower extremity vasculitis. In contrast, the study by Salvi and colleagues $(n=32)$ found superior efficacy of RTX (given either as 2 bi-weekly $1000 \mathrm{mg}$ infusions or as a single $500 \mathrm{mg}$ infusion) compared with IVCG (methylprednisolone $7.5 \mathrm{~g}$ total). The inactivation rate at 24 weeks in that study was $100 \%$ in the RTX group (with no reactivation noted) and $69 \%$ in the group receiving IVGC $(P<0.04)$. While reasons for the differences in RTX efficacy between the 2 studies remain unclear, it is possible that the longer mean disease duration of patients in the Stan study compared to the Salvi study (1 year $v$ s. 4.5 months, respectively) impacted the results. Until further study identifies subgroups of GO patients for whom RTX is a safe and effective therapy, this modality should not be considered first-line therapy, especially in patients with milder disease.

Orbital decompression surgery is generally reserved for patients with inactive GO needing rehabilitation to improve ocular function and quality of life or those with severe active disease and intolerance or insufficient response to immunosuppressive therapy ${ }^{[8]}$. Other indications include debilitating orbital or periorbital pain, excessive proptosis, orbital congestion, corneal exposure, deep orbital pain, dysthyroid optic neuropathy and enhanced cosmesis. While most patients benefit from decompression surgery, diplopia may develop or worsen in 10-50\%. Mild diplopia generally is improved by prisms inserted in the eyeglass lens. More significant impairment may require extraocular muscle surgery to obtain single vision in primary gaze.

It is clear that the currently available therapeutic options for Graves' hyperthyroidism and GO are inadequate; they are not uniformly successful and carry with them the potential for significant morbidity. Further, they target manifestations of the disease rather than pathophysiology. It is for these reasons that an active search for novel therapy targeting the unified cause of the clinical manifestations of the Graves' disease syndrome is underway. Studies performed by several laboratories have determined that the thyrotropin receptor is the primary target of the autoimmune process that occurs within the thyroid, orbit and skin in GD. Accordingly, therapies that target the function or immunogenicity of this receptor hold promise for unified treatment and prevention of the various clinical manifestations of the GD syndrome $e^{[\theta]}$.

\section{Conflict of Interest}

None declared.

\section{REFERENCES}

1. Bahn RS. Graves' ophthalmopathy. N Engl J Med 2010; 362:726-38.

2. Bartley GB. The epidemiologic characteristics and clinical course of ophthalmopathy associated with autoimmune thyroid disease in Olmsted County, Minnesota. Trans Am Ophthalmol Soc 1994; 92:477-588.

3. Bahn RS, Burch HB, Cooper DS, Garber JR, Greenlee MC, Klein I, et al. Hyperthyroidism and other causes of thyrotoxicosis: management guidelines of the American Thyroid Association and American Association of Clinical Endocrinologists. Endocr Pract 2011; 17:456-520.

4. Bartalena L, Baldeschi L, Dickinson AJ, Eckstein A, Kendall-Taylor P, Marcocci C, et al. Consensus statement of the European group on Graves' orbitopathy (EUGOGO) on management of Graves' orbitopathy. Thyroid 2008; 18:333-46.

5. Zang S, Ponto KA, Kahaly GJ. Intravenous glucocorticoids for Graves' orbitopathy: efficacy and morbidity. J Clin Endocrinol Metab 2011; 32032.

6. Stan MN, Garrity JA, Carranza Leon BG, Prabin T, Bradley EA, Bahn RS. Randomized controlled trial of rituximab in patients with Graves' orbitopathy. J Clin Endocrinol Metab 2015; 100:432-41.

7. Salvi M, Vannucchi G, Curro N, Campi I, Covelli D, Dazzi D. Efficacy of B-cell targeted therapy with rituximab in patients with active moderate to severe Graves' orbitopathy: a randomized controlled study. J Clin Endocrinol Metab 2015;100:422-31.

8. Dolman PJ. Evaluating Graves' orbitopathy. Best Pract Res Clin Endocrinol Metab 2012;26:229-48.

9. Bahn RS. Emerging pharmacotherapy for treatment of Graves' disease. Expert Rev Clin Pharmacol 2012;5:605-7.

How to cite this article: Bahn RS. Graves' disease syndrome: A need for unified therapy. J Transl Intern Med 2016; 4: 61-3. 